\title{
Atrioventricular block with coronary sinus potential dissociation after lateral mitral isthmus block: What is the mechanism?
}

\author{
Yosuke Nakatani ${ }^{1}$, Philipp Krisai ${ }^{2}$, Takashi Nakashima ${ }^{3}$, Romain Tixier $^{4}$, Nicolas Welte ${ }^{5}$, \\ Josselin Duchateau ${ }^{4}$, and Thomas Pambrun ${ }^{6}$ \\ ${ }^{1}$ University of Toyama \\ ${ }^{2}$ University of Bordeaux \\ ${ }^{3}$ 1. Electrophysiology and Ablation Unit and L'Institut de rythmologie et modélisation \\ cardiaque (LIRYC) \\ ${ }^{4}$ Centre Hospitalier Universitaire de Bordeaux \\ ${ }^{5}$ Centre Hospitalier Universitaire de Bordeaux Hôpital Haut-Lévêque \\ ${ }^{6}$ Hopital du Haut Leveque/LIRYC, Bordeaux
}

November 16, 2020

\section{EP Rounds}

Atrioventricular block with coronary sinus potential dissociation after lateral mitral isthmus block: What is the mechanism?

Yosuke Nakatani, MD, PhD; Philipp Krisai, MD; Takashi Nakashima, MD, PhD; Romain Tixier, MD; Nicolas Welte, MD; Josselin Duchateau, MD; Thomas Pambrun, MD

Department of Cardiac Pacing and Electrophysiology, IHU Liryc, Electrophysiology and Heart Modeling Institute, Univ. Bordeaux, Bordeaux University Hospital (CHU), 33600 Pessac- Bordeaux, France

Short running title: AV block with coronary sinus potential dissociation

Funding: YN is supported by the JHRS-EHRA Fellowship. PK is supported by the University of Basel, the Mach-Gaensslen foundation, and the Bangerter-Rhyner foundation.

Disclosures: None for any authors

Address for correspondence: Dr. Yosuke Nakatani, Department of Cardiac Pacing and Electrophysiology, Hôpital Cardiologique du Haut-Lévêque Avenue de Magellan, 33604, Bordeaux-Pessac, France. Telephone: +33-5-57656542; Fax: +33-5-57656509; E-mail: yosuke3gbst@gmail.com

\section{KEYWORDS}

Atrial tachycardia; Catheter ablation; Atrioventricular block; Vein of Marshall; Alcohol ablation; Coronary sinus

\section{CASE PRESENTATION}

A 61-year-old man was referred to our institution for ablation of an atrial tachycardia (AT) occurring after previous ablation of atrial fibrillation $(\mathrm{AF})$. The earlier procedure consisted of pulmonary vein isolation and cavotricuspid isthmus (CTI) ablation along with elimination of complex fractionated atrial electrograms (CFAEs) located at the roof and lateral wall of the left atrium, within the coronary sinus (CS), and at both sides of the interatrial septum. Baseline surface electrocardiogram showed sinus rhythm and first-degree 
atrioventricular (AV) block with a PR interval of $360 \mathrm{~ms}$ (Figure 1A). A decapolar catheter was positioned in the CS, and a quadripolar catheter in the left atrial appendage (LAA). Intracardiac recording revealed that: (1) LAA potentials preceded CS potentials, which exhibited a distal-to-proximal activation sequence (Figure 1B); (2) all pulmonary veins were still isolated; (3) CTI was still blocked, and (3) a line of block was observed at the left atrial roof. The clinical AT (Figures 1C and 1D) with the cycle length of $300 \mathrm{~ms}$ was induced by programmed atrial stimulation. High density mapping and post-pacing intervals were consistent with a localized reentry utilizing the vein of Marshall (VOM) epicardially and the mitral isthmus endocardially (Figure 1E). Ethanol was infused to the VOM as previously described ${ }^{1}$ and resulted in immediate AT termination. Bidirectional lateral mitral isthmus block was then confirmed by differential pacing maneuver. However, a transitory and complete AV block with a junctional escape rhythm spontaneously occurred (Figure 2A). For the duration of the complete AV block, LAA potentials consistently corresponded to $\mathrm{P}$ waves, and ventricular potentials constantly preceded CS potentials, which had no correlation with $\mathrm{P}$ waves (Figure 2B). These findings suggested that the LAA and the CS were activated by the sinus rhythm and the junctional rhythm, respectively. After the recovery of the AV conduction, the CS exhibited a proximal-todistal activation sequence (Figure $2 \mathrm{C}$ ). What is the mechanism of AV block with CS potential dissociation after the creation of a block line at the lateral mitral isthmus?

\section{DISCUSSION}

To investigate the underlying mechanism, bi-atrial activation was mapped in sinus rhythm (Figures $3 \mathrm{~A}$ and 3B). Both sides of the interatrial septum were transected by an incomplete line of block between the fossa ovalis and the CS. While one end of this line joined a posterior scar contiguous to the inferior vena cava (at the right side of the septum) and the right pulmonary vein isolation line (at the left side of the septum), a narrow isthmus of residual slow conduction was located at the annular end of the line. Initial PR prolongation was, therefore, due to a conduction slowing through the interatrial septum. As the CTI and roof lines were blocked, the sinus node activation propagated to the left atrium via the Bachman bundle, then in a clockwise direction around the mitral annulus through the lateral mitral isthmus, then to the AV node through the CS which exhibited a distal-to-proximal sequence, and finally collided with the delayed activation of the interatrial septum (Figure 3C).

After VOM ethanol infusion blocked the lateral mitral isthmus, the narrow isthmus at the interatrial septum remained as the only path for electrical propagation from the sinus node to the AV node (Figure 3D). We hypothesized that electrical conduction of this narrow isthmus was fragile enough to be temporarily blocked. Thus, the supra-nodal complete AV block observed in the current procedure was most likely due to a complete isolation of the lower parts of both atria. This isolation resulted in a complete dissociation of the sinus rhythm and the junctional rhythm, which separately activated the LAA and the CS, respectively (Figure 2B).

Although the interatrial septum is a frequent target of CFAE ablation, this will rarely result in an AV block $^{2,3}$ unless coexisting linear lesions disrupt the alternative conduction paths from the sinus node to the AV node. Given the high incidence of CTI-, roof-, and mitral-dependent flutters during a follow up after $\mathrm{AF}$ ablation, linear lesions are frequently created in the recurrent procedure. However, cautious analysis of interatrial conduction is needed before creating linear lesions in the CTI, roof, and mitral isthmus, when extensive CFAE ablation was previously performed at the interatrial septum. Furthermore, extensive ablation of the interatrial septum should probably be avoided to respect physiological atrial activation and to spare associated contractile function.

In the present case, a dual-chamber pacemaker was implanted before patient discharge.

\section{REFERENCES}

1. Pambrun T, Denis A, Duchateau J, Sacher F, Hocini M, Jaïs P, Haïssaguerre M, Derval N. MARSHALL bundles elimination, Pulmonary veins isolation and Lines completion for ANatomical ablation of persistent atrial fibrillation: MARSHALL-PLAN case series. J Cardiovasc Electrophysiol 2019;30:7-15. 
2. Verma A, Sanders P, Champagne J, Macle L, Nair GM, Calkins H, Wilber DJ. Selective complex fractionated atrial electrograms targeting for atrial fibrillation study (SELECT AF): a multicenter, randomized trial. Circ Arrhythm Electrophysiol 2014;7:55-62.

3. Hayward RM, Upadhyay GA, Mela T, Ellinor PT, Barrett CD, Heist EK, Verma A, Choudhry NK, Singh JP. Pulmonary vein isolation with complex fractionated atrial electrogram ablation for paroxysmal and nonparoxysmal atrial fibrillation: A meta-analysis. Heart Rhythm 2011;8:994-1000.

\section{FIGURES}

Figure 1

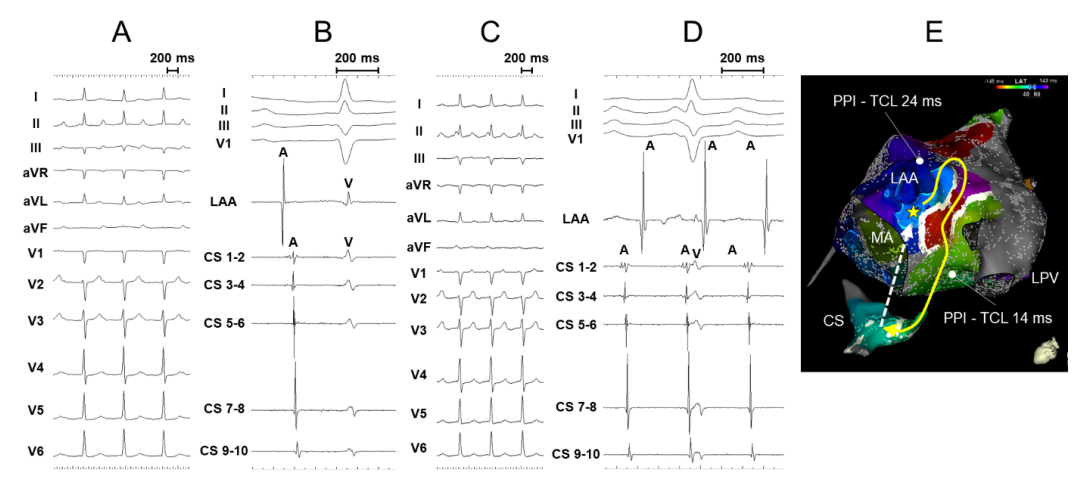

Figure 1 A: Surface electrocardiogram ( ECG) at baseline. B: Intracardiac ECG at baseline. C: Surface ECG during AT. D: Intracardiac ECG during atrial tachycardia (AT). E: The activation map during AT. Yellow arrows indicate the suspected tachycardia circuit. CS, coronary sinus; LAA, left atrial appendage; LPV, left pulmonary vein; MA, mitral annulus; PPI, post pacing interval; TCL, tachycardia cycle length.

Figure 2

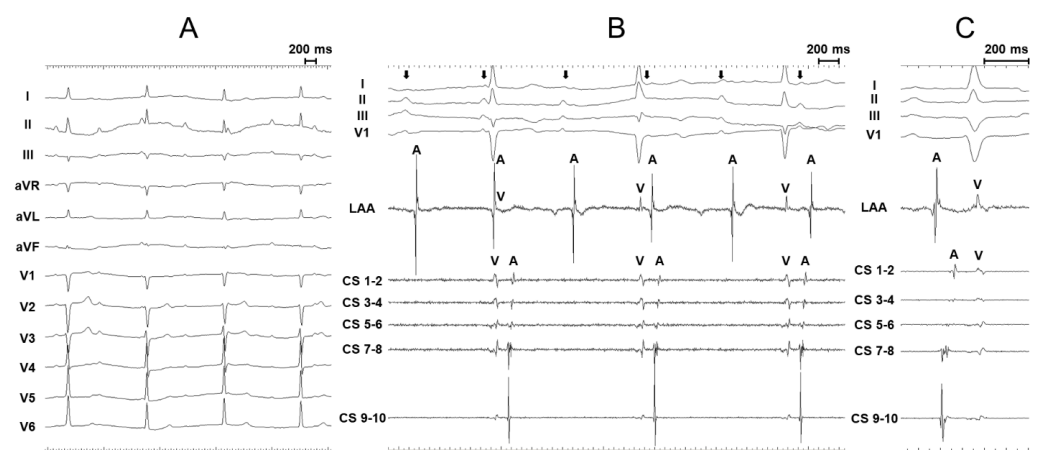

Figure 2 A:Surface electrocardiogram (ECG) after the ethanol infusion to the vein of Marshall (VOM). B: Intracardiac ECG after the ethanol infusion to VOM. Arrows indicate P waves. Note that P waves preceded atrial potentials in the LAA, and ventricular potentials preceded atrial potentials in the CS. C: Intracardiac ECG after the restoration of atrioventricular conduction. CS, coronary sinus; LAA, left atrial appendage. 
Figure 3
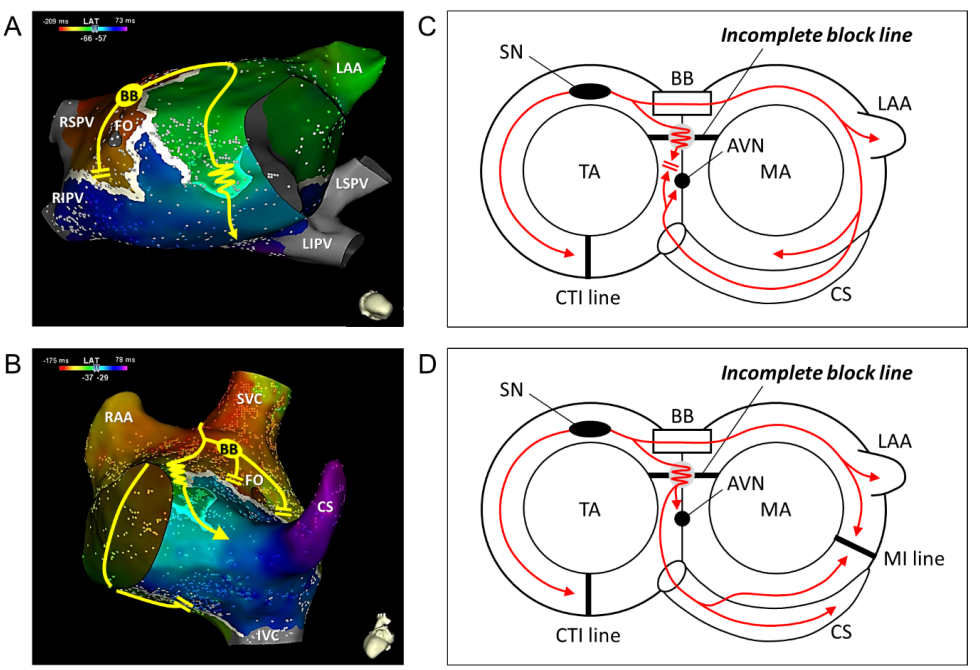

Figure 3 The activation maps of the left atrium (A ) and right atrium (B ). Electrical propagation is indicated by yellow arrows. An incomplete block line (white line) was observed at both sides of the interatrial septum, and a narrow isthmus was located at its annular end. The schema of the electrical activation in the atria before $(\mathbf{C})$ and after $(\mathbf{D})$ the ethanol infusion to the vein of Marshall (VOM). A gray area indicates a slow conduction area. AVN, atrioventricular node; BB, Bachman bundle; CS, coronary sinus; CTI, cavotricuspid isthmus; FO, fossa ovalis; IVC, inferior vena cava; LAA, left atrial appendage. LIPV, left inferior pulmonary vein; LSPV, left superior pulmonary vein; MA, mitral annulus; MI, mitral isthmus; RAA, right atrial appendage; RIPV, right inferior pulmonary vein; RSPV, right superior pulmonary vein; SN, sinus node; SVC, superior vena cava; TA, tricuspid annulus. 